
Not for reproduction, distribution or commercial use.

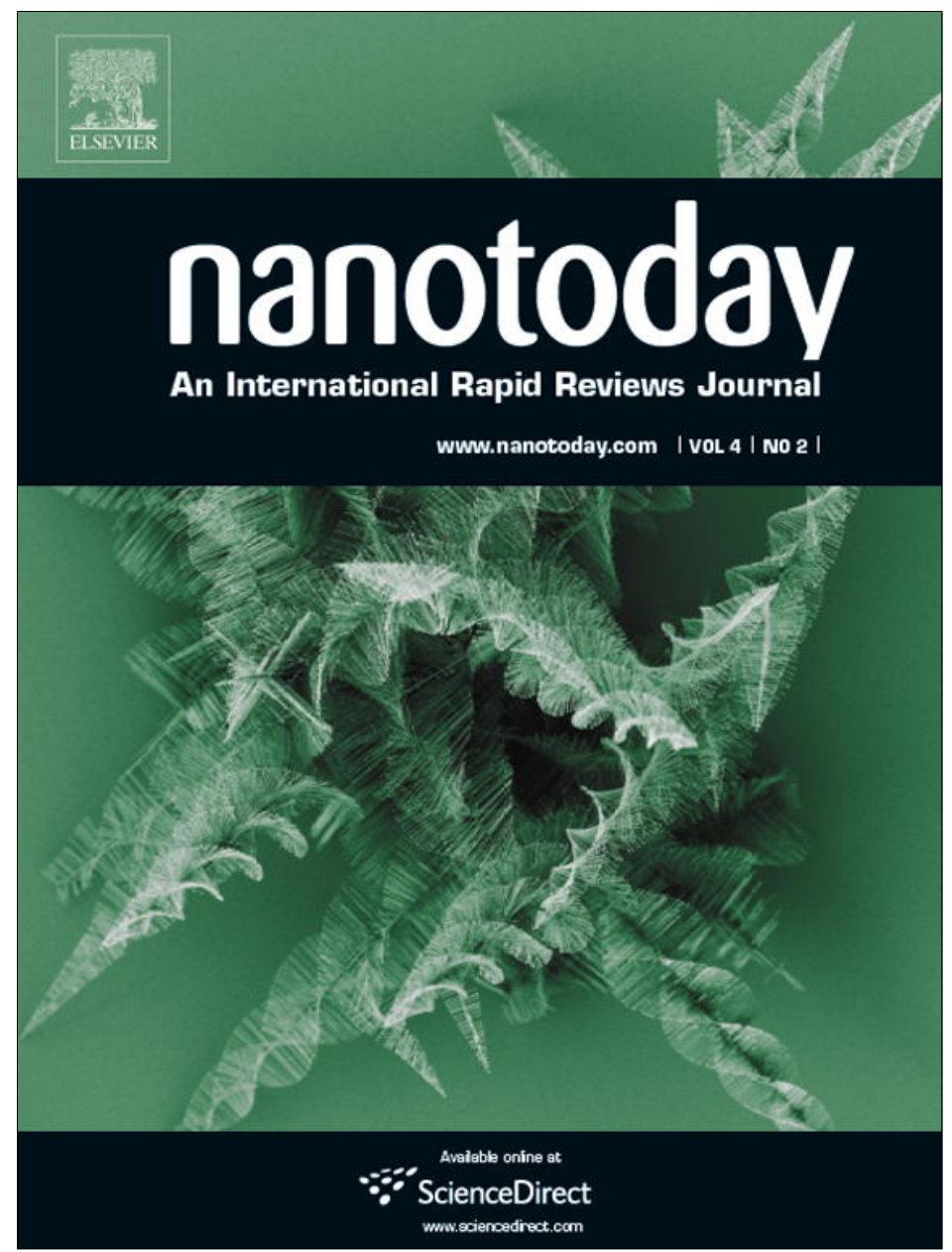

This article appeared in a journal published by Elsevier. The attached copy is furnished to the author for internal non-commercial research and education use, including for instruction at the authors institution and sharing with colleagues.

Other uses, including reproduction and distribution, or selling or licensing copies, or posting to personal, institutional or third party websites are prohibited.

In most cases authors are permitted to post their version of the article (e.g. in Word or Tex form) to their personal website or institutional repository. Authors requiring further information regarding Elsevier's archiving and manuscript policies are encouraged to visit:

http://www.elsevier.com/copyright 


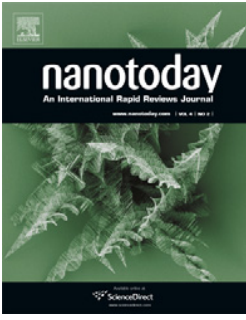

\title{
Simultaneous non-invasive analysis of DNA condensation and stability by two-step QD-FRET
}

\author{
Hunter H. Chen a,d,1, Yi-Ping Ho ${ }^{b, d, 1}$, Xuan Jiang ${ }^{c}$, Hai-Quan Maoc, \\ Tza-Huei Wang ${ }^{\mathrm{b}, *}$, Kam W. Leong ${ }^{\mathrm{d}, *}$
}

\author{
a Dept. of Biomedical Engineering, Johns Hopkins School of Medicine, Baltimore, MD, United States \\ b Dept. of Mechanical Engineering, Johns Hopkins University, Baltimore, MD, United States \\ c Dept. of Materials Science and Engineering, Johns Hopkins University, Baltimore, MD, United States \\ d Dept. of Biomedical Engineering, Duke University, 136 Hudson Hall, Box 90281, Duke University, Durham, \\ NC 27708, United States
}

Received 14 January 2009; received in revised form 10 February 2009; accepted 15 February 2009 Available online 10 March 2009

\section{KEYWORDS \\ Nanocomplex; \\ FRET; \\ Quantum dot; \\ Delivery; \\ Degradation; \\ Nonviral}

\begin{abstract}
Summary Nanoscale vectors comprised of cationic polymers that condense DNA to form nanocomplexes are promising options for gene transfer. The rational design of more efficient nonviral gene carriers will be possible only with better mechanistic understanding of the critical rate-limiting steps, such as nanocomplex unpacking to release DNA and degradation by nucleases. We present a two-step quantum dot fluorescence resonance energy transfer (twostep QD-FRET) approach to simultaneously and non-invasively analyze DNA condensation and stability. Plasmid DNA, double-labeled with QD (525 nm emission) and nucleic acid dyes, were complexed with Cy5-labeled cationic gene carriers. The QD donor drives energy transfer stepwise through the intermediate nucleic acid dye to the final acceptor Cy5. At least three distinct states of DNA condensation and integrity were distinguished in single particle manner and within cells by quantitative ratiometric analysis of energy transfer efficiencies. This novel two-step QDFRET method allows for more detailed assessment of the onset of DNA release and degradation simultaneously.

(c) 2009 Elsevier Ltd. All rights reserved.
\end{abstract}

\section{Introduction}

Nanoscale vectors in the form of cationic polymers complexed with plasmid DNA (pDNA) have emerged as safer, though less efficient, options for gene transfer compared to viruses [1]. The rational design of more efficient nonviral gene carriers will be possible only with better mechanistic understanding of the critical rate-limiting steps, such as nanocomplex unpacking to release the DNA cargo [2] and
* Corresponding authors at: Duke University, 136 Hudson
90281, Duke University, Durham, NC 27708, United States.

Tel.: +1 919660 8466; fax: +1 9196844488 .

E-mail addresses: thwang@jhu.edu (T.-H. Wang), kam.leong@duke.edu (K.W. Leong).

1 These authors contributed equally to this work. 
(a)

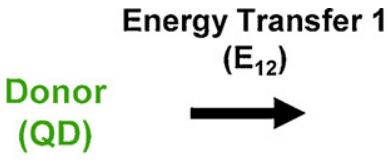

A

cceptor

(ND)
Energy Transfer 2

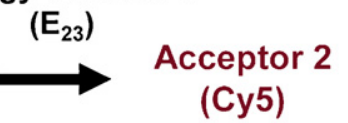

(b)

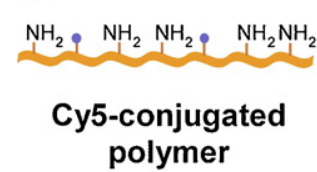

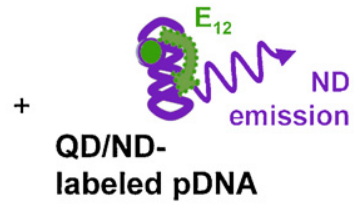

labeled pDNA

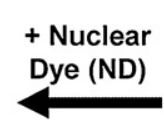

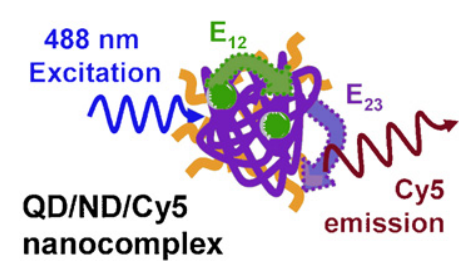

I

ND: ON

Cy5: ON
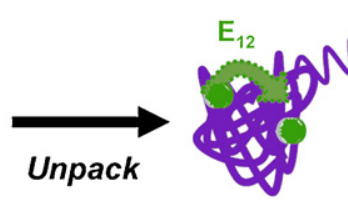

II

ND: ON

Cy5: OFF
QD-

labeled pDNA
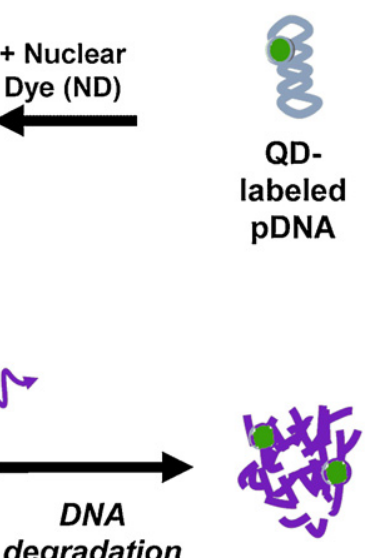

III

ND: OFF

Cy5: OFF

Figure 1 (a) Schematic of two-step QD-FRET. Excitation of the QD donor drives stepwise energy transfer $\left(E_{12}\right)$ through the nuclear dye (ND) which serves as the first acceptor/donor (or relay) for energy transfer to the second acceptor Cy5 ( $\left.E_{23}\right)$. (b) Plasmid DNA is double-labeled with $Q D$ and nuclear dyes before complexation with a Cy5-labeled cationic polymer to form nanocomplexes by self-assembly. Three distinct states of plasmid DNA (pDNA): (I) condensed within a nanocomplex, (II) released and intact, and (III) degraded, are distinguished by relative ND and Cy5 emission and calculated $E_{12}$ and $E_{23}$ efficiencies.

subsequent DNA degradation [3,4]. Condensation of pDNA by cationic polymers protects it from enzymatic degradation, but either pre-mature dissociation or overly stable binding would be detrimental to transfection efficiency [5-7]. Conventional methods to study nanocomplex stability and DNA degradation do not accurately reflect intracellular microenvironments or conditions. For example, gel electrophoresis is a common assay for nanocomplex stability but introduces an external electromotive force. Degradation of DNA has been studied in cells using fluorescence in situ hybridization (FISH) which requires permeabilizing the cell membrane. As an alternative, fluorescence energy transfer (FRET) with organic fluorophores has been used to study the dissociation of pDNA from lipoplexes or polyplexes $[8,9]$ or the degradation of pDNA $[10,11]$.

Quantum dots (QDs) have emerged as more efficient FRET donors due to their resistance to photobleaching, broad absorption, and narrow symmetric emission spectra which reduce spectral cross-talk [12-14]. Previously, we demonstrated that single-step quantum dot-mediated FRET (QD-FRET), comprised of a QD donor $(605 \mathrm{~nm}$ emission) and Cy5 acceptor, is an ultrasensitive method to detect the release of DNA from the nanocomplex [15]. Using this method, we developed a quantitative model and observed that the kinetics and intracellular compartment where dissociation occurs have a significant impact on transfection efficiencies for different gene carriers due to potential enzymatic barriers such as nuclease-mediated DNA degra- dation [16]. The dependence of nanocomplex stability and its ability to protect DNA on the structure-function relationships of the gene carrier require an integrated sensing platform whereby both DNA release from nanocomplexes and subsequent DNA degradation can be simultaneously monitored.

Here, we demonstrate a novel approach to study both DNA condensation and stability in a simultaneous and noninvasive manner by two-step QD-FRET. The incorporation of QDs as energy transfer donors permits continuous tracking of both rate-limiting processes during gene delivery. Previous applications of stepwise FRET with three fluorophores were to increase the effective distance for energy transfer [17-19] or to study protein interactions only [20]. In our approach, a QD donor ( $525 \mathrm{~nm}$ emission) is paired with a nuclear dye (ND) and a second acceptor Cy5 for stepwise FRET. The relay ND serves a dual purpose: first, as the acceptor/donor (or relay) and second, as an indicator for DNA integrity. At least three states of pDNA can be distinguished through combinations of ND and Cy5 emission and quantitative ratiometric analysis of FRET efficiencies $[14,18]$. Two gene carriers, chitosan [21] and polyethylene glycol-polyphosphoramidate block copolymer (PEG-b-PPA) [22], were compared since both polymers elicit transgene expression in vitro and in vivo while forming nanocomplexes with contrasting structures and stabilities. Being amenable to single particle characterization, two-step QD-FRET is particularly valuable for understanding critical barriers to 

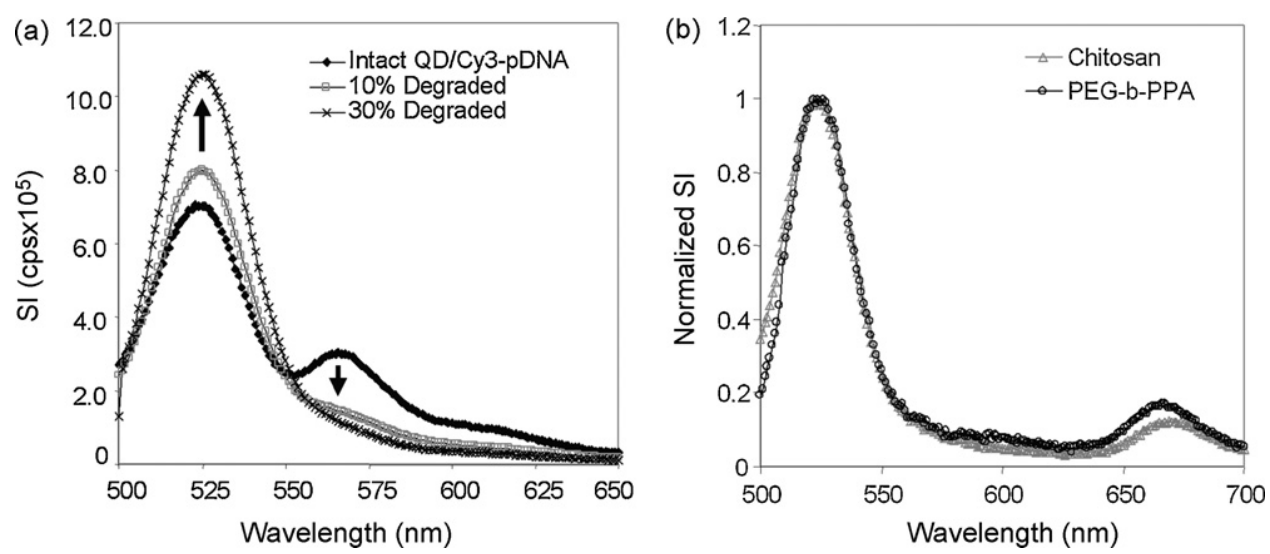

Figure 2 Spectral characterization of two-step QD-FRET. (a) Intact QD/Cy3-pDNA exhibited QD (525 nm) and $E_{12}$-mediated Cy3 $(570 \mathrm{~nm}$ ) emission $(\$)$. Increasing fractions of degraded QD/Cy3-pDNA: 10\% (gray $\square$ ) and 30\% $(\times$ ). Total pDNA content was kept constant. (b) Emission spectra of nanocomplexes comprised of QD/Cy3-pDNA and Cy5-labeled (670 nm) chitosan (gray $\triangle$ ) or PEG-bPPA $(\bigcirc)$ showed two-step energy transfer. The signal intensity here was normalized to the QD peak value at $525 \mathrm{~nm}$ to compare the energy transfer between the different nanocomplex polymer compositions.

gene transfer given the heterogeneity of nanocomplexes and microenvironments encountered within cells.

\section{Results and discussion}

\section{Versatility of two-step QD-FRET}

Our three-fluorophore FRET system is designed with a two-step energy transfer [23], henceforth named two-step QD-FRET. The QD donor ( $525 \mathrm{~nm}$ emission) drives energy transfer to an intermediate acceptor ND (energy transfer $E_{12}$ ) serving as a relay donor to the final acceptor Cy5 (energy transfer $E_{23}$ ) (Fig. 1a). Each fluorophore is selected based on their compatibility as single-step FRET pairs, where spectral overlap between the donor and acceptor is maximized and potential cross-talk between channels is minimized. Within this system, three main states of pDNA are indicated by combinations of FRET-mediated emission from the ND and/or Cy5 (Fig. 1b). (I) When pDNA, double-labeled with QD and ND (QD/ND-pDNA), are condensed into compact nanocomplexes, the QD donor drives energy transfer through the ND which acts as a relay to Cy5 conjugated on the polymeric gene carrier. For stable nanocomplexes, both the ND and Cy5 are 'on' or actively exhibiting intra- $\left(E_{12}\right)$ and intermolecular FRET $\left(E_{23}\right)$, respectively. (II) When the nanocomplex begins to unpack and release intact pDNA, only the ND is 'on' through $E_{12}$ while Cy5 is 'off' due to the loss of $E_{23}$. (III) The subsequent onset of degradation of free pDNA is indicated by the ND and Cy5 turning 'off' due to the loss of $E_{12}$ and $E_{23}$, respectively.

Two cyanine-based NDs having similar spectral properties but different DNA-binding mechanisms were compared to illustrate the versatility of two-step QD-FRET. An intercalating dye (BOBO-3) or covalently binding dye (Cy3) was used to label pDNA as intramolecular FRET acceptors for the QD donor (Supporting Information) [17,24]. Intact plasmids labeled with $Q D$ and $C y 3$ via a covalent linker (QD/Cy3pDNA) exhibited two corresponding emission peaks due to $E_{12}$ (Fig. 2a). Similarly, plasmids labeled with both QD and BOBO-3 (QD/BOBO-pDNA) exhibited two peaks corre- sponding to their emission maxima (Supporting Information, Fig. 1a). Subsequent experiments characterizing DNA degradation were conducted using QD/Cy3-pDNA due to the quenching of QDs caused by unbound BOBO-3 above nM concentrations (Supporting Information, Fig. 2). It is expected that this quenching effect may be mitigated by further optimization of staining ratios such that the concentration of unbound $\mathrm{BOBO}-3$ remains less than $\mathrm{nM}$ magnitudes while still allowing for sufficient energy transfer.

To demonstrate the versatility of this nanophotonics approach, two gene carriers, chitosan $[21,25]$ and PEG-b-PPA [22], which form contrasting nanocomplex structures were compared. When complexed with DNA, chitosan generates polyplex structures [25] while PEG-b-PPA forms core-shell micelles [22]. The QD/BOBO or QD/Cy3-labeled pDNA were stably complexed with either Cy5-labeled chitosan or PEGb-PPA. Labeling of the pDNA and polymer had minimal effect on the stability and physical properties of nanocomplexes, compared to unlabeled nanocomplexes (Supporting Information, Fig. 3). The spectra from these triple-labeled nanocomplexes showed that normalized signal intensities of Cy5 for chitosan and PEG-b-PPA were similar (Fig. 2b and Supporting Information, Fig. 1b). Hence, two-step QDFRET is a versatile platform that may be applied to various cationic gene carriers and nanocomplex morphologies.

\section{Quantifying DNA degradation by $E_{12}$}

To assess the sensitivity of $E_{12}$ efficiency to detect DNA degradation, intact QD/Cy3-pDNA were mixed with varying fractions of degraded QD/Cy3-pDNA while keeping the total pDNA amount constant. Increasing fractions of degraded QD/Cy3-pDNA resulted in higher QD signals and reduced FRET-mediated Cy3 signals (Fig. 2a). The QD and Cy3 signals varied linearly with increasing fractions of degraded pDNA as expected (Fig. $3 a$, inset). $E_{12}$ efficiencies were calculated from measured signal intensities according to Eq. (1), and an exponential decay function was fitted to experimentally measured $E_{12}$ efficiencies by least squares $\left(R^{2}=0.96\right)$ to generate the $E_{12}$ calibration curve: $y=0.09+0.95 e^{-0.13 x}$, where $y$ 

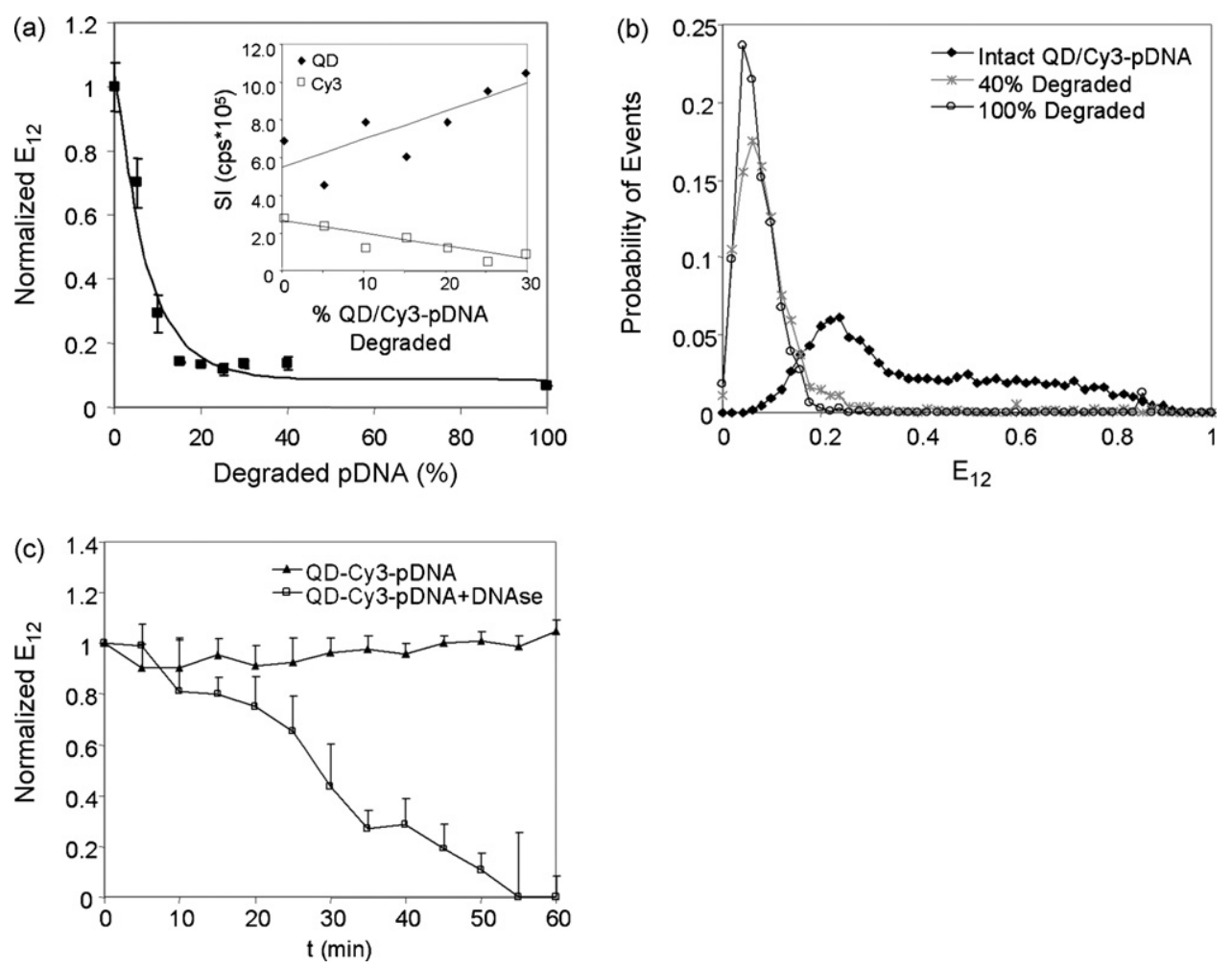

Figure 3 DNA degradation by QD-FRET. (a) Experimentally measured $E_{12}$ efficiencies ( $\mathbf{\square}$, mean $\pm \mathrm{SD}, n=3$ ) and the $E_{12}$ calibration curve: $y=0.09+0.95 e^{-0.13 x}$, where $y$ is the normalized $E_{12}$ and $x$ is the \% degraded pDNA. The QD ( $\left.\downarrow\right)$ and Cy3 signals ( $\left.\square\right)$ varied linearly with increased fractions of degraded pDNA as expected (inset). The non-linearity of the $E_{12}$ calibration curve was a result of the ratio used to calculate $E_{12}$ efficiency. (b) By single molecule detection, the probability histogram for $E_{12}$ efficiency values for completely intact QD/Cy3-pDNA ( $\diamond)$, a $40 \%$ mixture of degraded pDNA (*), and degraded pDNA only $(\bigcirc)$. (c) The $E_{12}$ efficiency of QD/Cy3-pDNA normalized to the initial value was monitored for $60 \mathrm{~min}$ with nucleases (DNase I) ( $\square$ ) and without nucleases ( $\Delta$ ) $($ mean $\pm S D, n=3)$.

is the normalized $E_{12}$ and $x$ is the \% degraded pDNA (Fig. 3a). The sensitivity range according to this $E_{12}$ calibration curve was up to $\sim 30 \%$ degraded pDNA, above which the $E_{12}$ value remained constant at $\sim 0.1$. The reduction in $E_{12}$ efficiency was caused by the diffusion of small DNA oligomers away from the QD donor as plasmid DNA was being cleaved by nucleases and thereby increasing donor-acceptor separation [26] and reducing the acceptor-donor ratio. Similar sensitivity for degradation was reported for siRNA [10] and DNA oligomers [11], illustrating the potential application of QD-FRET to other nucleic acid molecules.

The mixed population of coiled and relaxed forms of individual plasmids were resolved by the sensitivity of energy transfer and single molecule detection (SMD) (Fig. 3b) $[14,27]$. While nearly all intact DNA had $E_{12}$ values above 0.2 , the wide distribution of $E_{12}$ for intact pDNA reflected the mixed presence of coiled plasmids indicated by higher $E_{12}$ values and relaxed plasmids by lower $E_{12}$ values. In contrast, the $E_{12}$ distribution for degraded QD/Cy3-pDNA shifted to the left $\left(E_{12}<0.2\right)$ due to the loss of intramolecular FRET and was consistent with the $E_{12}$ calibration curve in Fig. $3 a$. These observations validate that FRET efficiencies obtained from the emission spectra and single molecule data were in strong agreement and suggest that $E_{12}$ values are well suited to signal the onset of DNA degradation as a bulk population or as single molecules.
The degradation kinetics of QD/Cy3-pDNA treated with nucleases showed that $E_{12}$ efficiency declined whereas the $E_{12}$ of the control QD/Cy3-pDNA (without nucleases) remained constant (Fig. 3c). At least 30\% of pDNA was degraded after $45 \mathrm{~min}$ where $E_{12}$ efficiencies declined below 0.2 . The resistance of the $Q D$ donor to photobleaching allowed continuous tracking of the status of QD-labeled pDNA for at least $1 \mathrm{~h}$.

\section{Single molecule and temporal analysis of DNA condensation and degradation}

Being amenable to single particle characterization, twostep QD-FRET is particularly valuable for understanding critical barriers to gene transfer given the heterogeneity of nanocomplexes and microenvironments encountered within cells. By three-color SMD (Supporting Information, Fig. 4) [28], the distributions of pDNA having distinct condensed or degraded states were detected. Stable QD/Cy3/Cy5-chitosan nanocomplexes exhibited more efficient intermolecular energy transfer between the DNA and polymer $\left(E_{23}>0.6\right)$ (Fig. 4b). Nanocomplexes treated with DNase I had nearly identical $E_{23}$ efficiency distributions as control nanocomplexes, suggesting that the gene carrier provided effective protection from enzymatic degradation. 

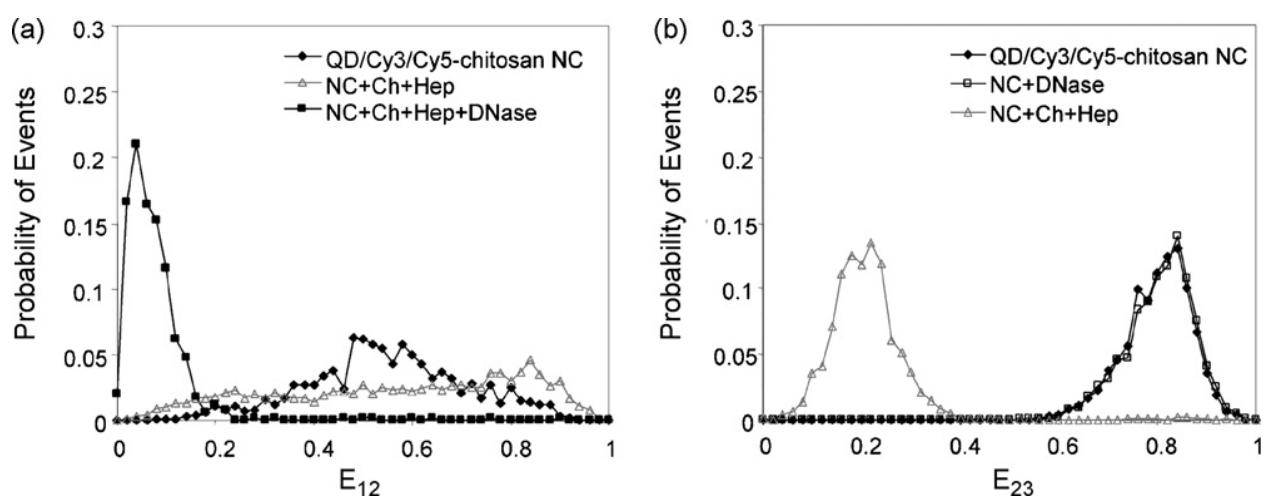

Figure 4 Characterization of two-step QD-FRET nanocomplexes by single molecule detection. The probability histograms of (a) $E_{12}$ efficiencies and (b) $E_{23}$ efficiencies for QD/Cy3/Cy5-chitosan nanocomplexes which were untreated ( $)$ ), treated with DNase I $(\square)$, disrupted with heparin (Hep) and chitosanase (Ch) (gray $\triangle$ ), or disrupted in combination with DNase I ( $\mathbf{\square})$.

Further, $E_{23}$ efficiencies above 0.6 indicated that chitosan remained stably complexed to pDNA after nuclease treatment. When treated with chitosanase and heparin for $1 \mathrm{~h}$ to disrupt the nanocomplexes, the $E_{23}$ efficiency distribution shifted down to below 0.4 , which was consistent with measured unpacking kinetics and bulk spectral measurements (Supporting Information, Fig. 5). The gradual decrease in $E_{23}$ of disrupted nanocomplexes was caused by the initial swelling or loosening of the nanocomplex which was followed by a more rapid decline after $30 \mathrm{~min}$ as it continued to unpack (Fig. 5b). The addition of nucleases along with chitosanase and heparin steepened the rate of $E_{23}$ reduction, indicating a faster unpacking of the nanocomplex. As the nanocomplexes were being disrupted, the added nucleases expedited the dissociation process since DNA was also susceptible to cleavage. Similarly to $E_{12}$ efficiency, the $E_{23}$ efficiency is sensitive to the separation between $\mathrm{Cy} 3$ and Cy5 during the unpacking process where the nanocomplex loosens as the pDNA and gene carrier continue to dissociate.

The integrity of encapsulated and released pDNA was maintained as indicated by their $E_{12}$ efficiencies being above 0.2 (Fig. 4a). When encapsulated within a nanocomplex, condensed pDNA showed a more uniform distribution of $E_{12}$ compared to that for free pDNA (Fig. 3b). Likewise, plasmids released from disrupted nanocomplexes also exhibited a wider $E_{12}$ distribution, which was attributed to their relaxation after release. Combining disruption with nucleases resulted in the degradation of released pDNA, which was indicated by the clear shift in $E_{12}$ efficiency distributions to below 0.2 (Fig. $4 a$ ) and the rapid decline of $E_{12}$ efficiency during the first $5 \mathrm{~min}$ followed by a steady decline to less than 0.2 after $40 \mathrm{~min}$ as more DNA was degraded (Fig. 5a). Taken together, these results confirmed that condensed DNA was protected by the gene carrier from nuclease-mediated degradation through steric hindrance [25], and more importantly, provided direct evidence that unpacking occurs prior to DNA cleavage by nucleases.

\section{Analyzing intracellular unpacking and DNA degradation}

Combining quantitative ratiometric FRET analysis with SMD may resolve subpopulations of nanocomplexes and DNA having $E_{23}$ and $E_{12}$ values that signify intermediate stages of unpacking and degradation, respectively. These intermediate stages can be correlated to energy transfer efficiencies calculated from images of transfected cells, giving a more accurate description of unpacking and DNA degradation kinetics within cells. HEK293 cells were transfected with
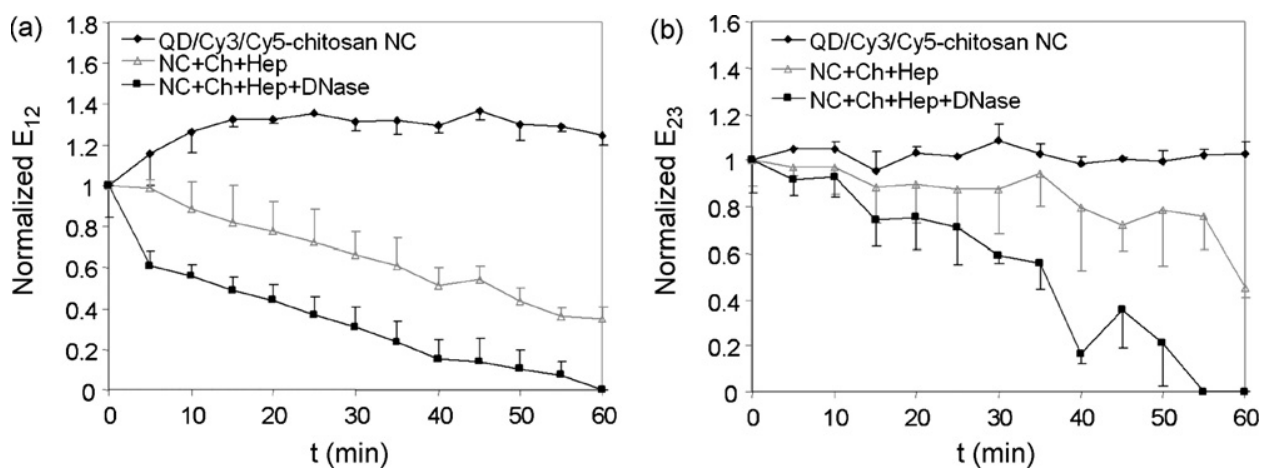

Figure 5 Temporal characterization of two-step QD-FRET nanocomplexes. (a) Changes in $E_{12}$ efficiencies for DNA degradation and (b) $E_{23}$ efficiencies for dissociation of QD/Cy3/Cy5-chitosan nanocomplexes (NC) were monitored for 60 min. Nanocomplexes were untreated $(\downarrow)$ or disrupted by treatment with heparin (Hep) and chitosanase $(\mathrm{Ch})$ (gray $\triangle$ ), or disrupted in combination with DNase $I(\boldsymbol{\square})$. Efficiencies were normalized to the initial value before treatment (mean $\pm S D, n=3$ ). 
triple-labeled chitosan and PEG-b-PPA nanocomplexes to study the intracellular release and degradation of pDNA by two-step QD-FRET. At least three distinct states of pDNA were observed by calculating $E_{12}$ and $E_{23}$ efficiencies on a pixel-by-pixel basis using Eqs. (1) and (2), respectively (Supporting Information). At $4 \mathrm{~h}$ post-transfection, chitosan nanocomplexes remained stable or began dissociating since $E_{23}$ efficiencies were below 0.6 (Fig. 6a). As a result, most of the pDNA associated with chitosan maintained its integrity since their $E_{12}$ efficiencies were above 0.5 in value. In contrast, nearly all the pDNA were dissociated from PEG-b-PPA micelles as indicated by $E_{23}$ efficiencies below 0.4 (Fig. 6b).
The free pDNA showed a range of $E_{12}$ efficiencies but some pDNA clearly have $E_{12}$ efficiencies less than 0.2 , indicating that these plasmids were degraded as a consequence of being rapidly released. Microinjected pDNA were reported to be degraded within $2 \mathrm{~h}[3,4,29]$. The unpacking kinetics observed was consistent for chitosan [16] and PEG-b-PPA, which formed relatively less stable complexes (unpublished data). The PEG conjugation may increase the colloidal stability of PEG-b-PPA micelles but reduce the complex stability of the micelles [11]. These results underscore the delicate balance between maintaining stability to preserve DNA and allowing for its release to increase bioavailability. (a)
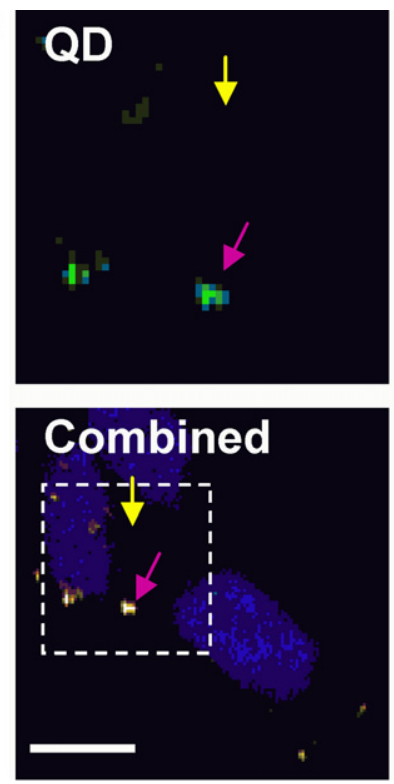

(b)
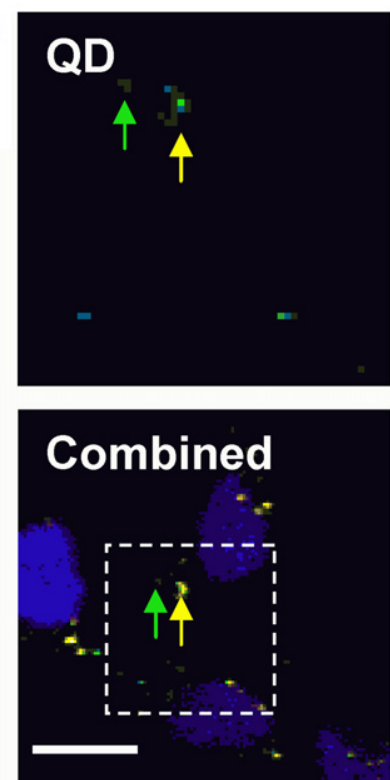
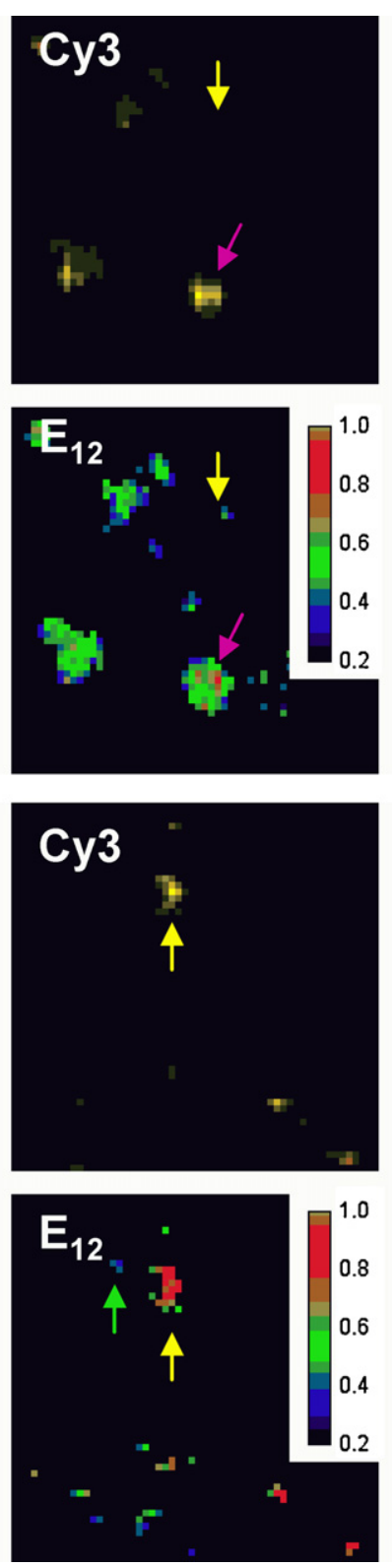
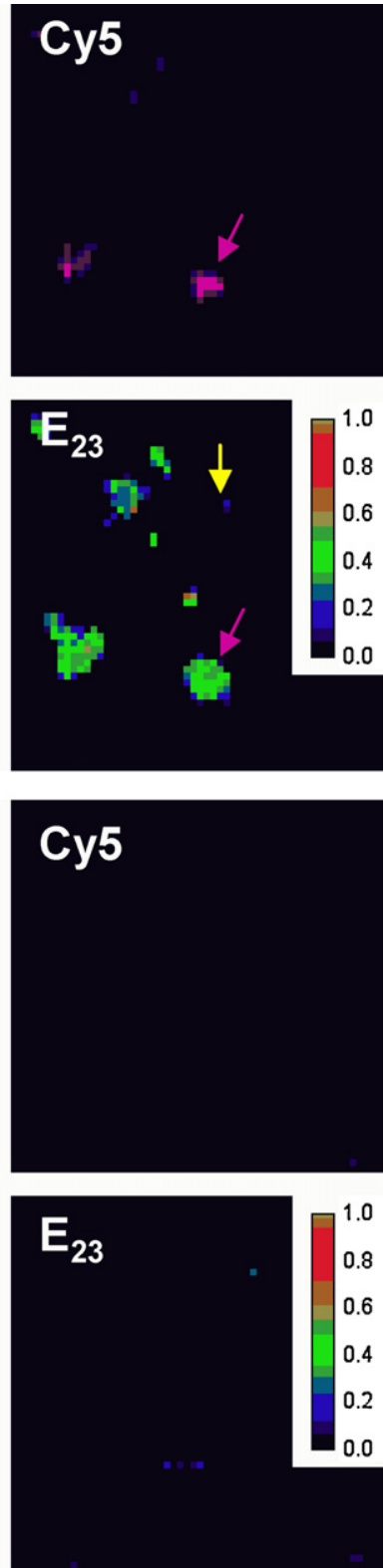

Figure 6 Intracellular analysis by two-step QD-FRET. HEK293 cells were transfected with (a) QD/Cy3/Cy5-chitosan and (b) $\mathrm{QD} / \mathrm{Cy} 3 /$ Cy5-PEG-b-PPA nanocomplexes. The combined image of QD, Cy3, and Cy5 channels at $4 \mathrm{~h}$ post-transfection shows colocalized signals relative to the nucleus (blue). Individual QD (green), Cy3 (yellow), Cy5 (purple) channels, $E_{12}$ efficiencies, and $E_{23}$ efficiencies are shown for the region of interest (dashed box). Energy transfer efficiencies for DNA degradation $\left(E_{12}\right)$ and unpacking $\left(E_{23}\right)$ were calculated on a pixel-by-pixel basis and their values are indicated by the pseudo-color scale. Examples of condensed pDNA within nanocomplexes (purple arrow), released intact pDNA (yellow arrow), and degraded pDNA (green arrow) are shown. Scale bar $=10 \mu \mathrm{m}$. 


\section{Discussion}

We have demonstrated a two-step QD-FRET approach to simultaneously and non-invasively monitor DNA condensation and integrity. The use of QDs as the donor allowed efficient stepwise energy transfer to a relay ND and acceptor Cy5. At least three distinct states of pDNA were distinguished by FRET-mediated emission from the ND and/or Cy5 and by quantitative ratiometric analysis of $E_{12}$ or $E_{23}$ efficiencies. After considering applicable quantification methods [30-32], the ratiometric analysis presented here was chosen to be the most meaningful method to compare data across different detection platforms used in this study: spectrofluorometry, 3-channel SMD, fluorescence microplate reader, and confocal microscopy. We obtained similar results across these platforms, suggesting that the ratiometric analysis was an appropriate metric in the single [14] and two-step QDFRET systems to monitor DNA integrity and condensation.

Condensed, free and intact, or partially degraded pDNA were identified in acellular and intracellular conditions. Given the Forster distances for the QD/Cy3 pair and Cy3/Cy5 pair were $5.8 \mathrm{~nm}$ [24] and $6.0 \mathrm{~nm}$ [28], respectively, the high sensitivity of energy transfer to changes in donor-acceptor distance and ratios allowed various stages of unpacking and degradation to be detected. The $E_{12}$ efficiency is sensitive to the diffusion of small oligomers away as DNA was being cleaved which reduced the acceptor-donor ratio. Similarly, $E_{23}$ is sensitive to the separation between the pDNA and gene carriers as they dissociate. There is also a possibility that the QD and Cy5 may participate in energy transfer [23], but any such energy transfer is expected to be minimal due to the small spectral overlap. Any effect of QD-Cy5 energy transfer $\left(E_{13}\right)$ is expected to be negligible in our analysis since $E_{13}$ would contribute only in the case of compact nanocomplexes. Further, the narrow emission spectra of QDs allowed efficient energy transfer mainly through the intermediate ND to the final acceptor Cy5. Intercalated or covalently bound nuclear dyes were both compatible relays within this versatile two-step QD-FRET system. Unlike previous reports which have used relay fluorophores solely to increase the effective distance range for QD-FRET [17], the $\mathrm{Cy} 3$ in this system serves as both a relay fluorophore as well as an indicator of DNA integrity by intramolecular FRET.

This versatility was again demonstrated by applying twostep QD-FRET to analyze different polymers. Chitosan and PEG-b-PPA formed nanocomplexes with contrasting structures and unpacking rates which are factors that play a role in determining eventual transfection efficiency. Considering that the mean luciferase expression levels were at least $10 \times$ lower for chitosan than PEG-b-PPA in hepatocytes at $6 \mathrm{~h}$ post-transfection $(0.02$ and $0.36 \mathrm{ng}$ luciferase/mg protein, respectively) and $48 \mathrm{~h}$ post-transfection $[22,33]$, the intracellular analysis of $E_{12}$ and $E_{23}$ suggested that the slower unpacking of chitosan nanocomplexes may have preserved DNA integrity but ultimately hampered its ability to release DNA, reducing its intracellular bioavailability. In contrast, PEG-b-PPA micelles dissociated readily in cells, rendering DNA accessible for transcription but also susceptible to degradation which had less of an impact on transfection efficiency in this case. From this comparison, two-step QD-FRET resolved mechanistic differences in the cellular processing of different gene carriers and identified the relative impor- tance of unpacking and protection specific to individual carriers and their effect on short- and long-term transgene expression.

In conclusion, two-step QD-FRET is a sensitive and versatile platform to study rate-limiting barriers to efficient gene transfer. To our knowledge, this is the first report where DNA dissociation from nanocomplexes and subsequent DNA degradation may be tracked in a simultaneous and noninvasive manner. This is also the first attempt in using stepwise QD-FRET as a sensing system to identify various states of DNA. Quantitative ratiometric analysis of energy transfer efficiencies was correlated to at least three distinct states of DNA condensation and integrity. Condensed, free and intact, or partially degraded pDNA were identified in a single particle manner and within cells. Different nuclear dyes and gene carriers were compared to demonstrate the potential applicability of this method to other types of vectors. Thus, two-step QD-FRET is poised to expedite the design of more efficient vectors for nanomedicine by offering a more accurate mechanistic understanding of critical barriers to gene delivery.

\section{Experimental}

\section{Labeling of plasmid DNA and polymeric gene carriers}

Plasmid DNA (pEGFP-C1, $4.9 \mathrm{~kb}$, Clontech, Mountain View, CA) was labeled with nuclear dyes and streptavidinfunctionalized QD525 (Qdot 525 ITK, Invitrogen, Carlsbad, CA) as described previously [15]. Briefly, pDNA were biotinylated as described by the manufacturer (Label IT Biotin, Mirus Bio, Madison, WI) but scaled to have $\sim 1-2$ biotin labels per pDNA. The number of QDs labeled onto each pDNA is estimated to be $\sim 1-3$ [15]. Prior to QD-labeling, biotinylated plasmid was labeled with either one of two cyanine-based nuclear dyes which have different DNA-binding mechanisms but similar spectral characteristics for comparison. An intercalating dye, BOBO-3 (Invitrogen, Carlsbad, CA), and a covalently binding dye, Cy3 (Label IT Cy3, Mirus Bio, Madison, WI), were selected based on their excitation and emission spectra to allow for efficient energy transfer $[17,24]$. The primary amines of the cationic polymeric gene carriers were labeled with $\mathrm{N}$-hydroxy-succinimidefunctionalized Cy5 (Amersham Biosciences, Piscataway, NJ) through carbodiimide chemistry. A solution of Cy5 dye was gradually added to aqueous solutions of chitosan $(390 \mathrm{kDa}$, 83.5\% deacetylated, Vanson, Redmond, WA) or PEG-b-PPA (synthesized as described in [22]) and stirred continuously for at least $12 \mathrm{~h}$. For chitosan, the reaction mixture was maintained at $\mathrm{pH} \sim 6.5$ to keep chitosan soluble. Any remaining free dye was removed by dialysis to obtain only Cy5-labeled polymer. See Supporting Information for full details on labeling.

\section{Synthesis of two-step QD-FRET nanocomplexes}

The QD/BOBO-pDNA or QD/Cy3-pDNA was electrostatically complexed with a Cy5-labeled chitosan or PEG-b-PPA to form triple-labeled nanocomplexes (QD/ND-pDNA/Cy5-polymer). Nanocomplexes were prepared from chitosan as described 
previously [15]. Briefly, Cy5-chitosan (pH 5.5-5.7; 0.1\% in $25 \mathrm{mM}$ acetic acid solution) and QD-labeled pDNA in $50 \mathrm{mM}$ of sodium sulfate solution were both heated to $55^{\circ} \mathrm{C}$ separately. Equal volumes of both solutions were quickly mixed under high vortexing. Chitosan nanocomplexes were formed at an N/P ratio (primary amine to phosphate ratio) of $4: 1$. The synthesis of nanocomplexes formed with PEG-b-PPA are described elsewhere [22]. Briefly, pDNA was added to an aqueous solution of PEG-b-PPA. Complexes were formed at an N/P ratio of $8: 1$ by adding equal volumes of polymer solution to the DNA solution and then vigorously mixed. All polyplexes were then used without further purification. Fluorescence emission spectra of QD/ND/Cy5-nanocomplexes were scanned by a spectrofluorometer (Spex FluoroLog-3, Horiba Jobin Yvon, Edison, NJ). The excitation wavelengths for $\mathrm{QD} / \mathrm{BOBO}-3$ and $\mathrm{QD} / \mathrm{Cy} 3$-labeled nanocomplexes were $425 \mathrm{~nm}$ and $405 \mathrm{~nm}$, respectively. See Supporting Information for full details on characterizing polyplex size and zeta potential.

\section{Ratiometric analysis of FRET efficiencies}

A ratiometric method [14,18] was used to calculate efficiencies for energy transfers between the QD and ND $\left(E_{12}\right)$ and between the ND and Cy5 $\left(E_{23}\right)$, separately, according to following equations:

$$
\begin{aligned}
& E_{12}=\frac{I_{\mathrm{ND}}}{I_{\mathrm{QD} 525}+I_{\mathrm{ND}}} \\
& E_{23}=\frac{I_{\mathrm{Cy} 5}}{I_{\mathrm{ND}}+I_{\mathrm{Cy} 5}}
\end{aligned}
$$

where $I_{\mathrm{QD} 525}, I_{\mathrm{ND}}$, and $I_{\mathrm{Cy} 5}$ represent the burst or signal intensities obtained from each corresponding channel and ND is either BOBO-3 or Cy3.

\section{Disruption of nanocomplexes and degradation of DNA}

QD/Cy3-pDNA was degraded with DNase I (2 U/ (g pDNA; New England Biolabs, Ipswich, MA) for $1 \mathrm{~h}$ at $37^{\circ} \mathrm{C}$. To inhibit DNase I, $10 \%$ by volume of $400 \mathrm{mM}$ TAE buffer supplemented with $10 \mathrm{mM}$ EDTA was added. While keeping the total DNA amount constant per sample, mixtures of degraded and intact QD/Cy3-pDNA were prepared and its emission spectra scanned with an excitation at $405 \mathrm{~nm}$. The calibration curve relating $E_{12}$ efficiency according to Eq. (1) to the fraction of degraded pDNA was determined by least squares fitting of an exponential function to the experimental $E_{12}$ data normalized to $E_{12}$ value of intact QD/Cy3-pDNA (Origin v6.0, OriginLab, Northampton, MA). The kinetics of QD/Cy3/Cy5chitosan nanocomplex dissociation and DNA degradation were monitored for $1 \mathrm{~h}$ at $37^{\circ} \mathrm{C}$. Triplicate wells containing QD/Cy3/Cy5-chitosan nanocomplexes were treated with heparin sulfate $(8 \mu \mathrm{g} / \mu \mathrm{g}$ pDNA; Sigma-Aldrich, St. Louis, MO) and chitosanase ( $14 \mu \mathrm{g}$ enzyme/ $\mu$ g polymer; Seikagaku America, Ijamsville, MD) and/or DNase I ( $2 \mathrm{U} / \mu \mathrm{g}$ pDNA). FRET efficiencies $E_{12}$ and $E_{23}$ were calculated according to Eqs. (1) and (2), respectively, and normalized to the initial value. See Supporting Information for full details on data acquisition.

\section{3-Channel single molecule detection}

The custom setup used for single molecule detection is similar to the one reported previously [15]. Energy transfer efficiencies were analyzed and distributions of $E_{12}$ and $E_{23}$ were obtained for each 100 -s measurement. The probability histogram, based on the distributions of $E_{12}$ and $E_{23}$, was then normalized according to the number of total events to keep the sum of probabilities (area under the curve) for each histogram equal to unity and plotted from the average of three independent measurements. See Supporting Information for full details on data acquisition.

\section{Confocal imaging}

HEK293 cells (ATCC, Manassas, VA) were transfected with QD-FRET polyplexes containing $0.5 \mu \mathrm{g}$ DNA in $0.5 \mathrm{ml}$ of reduced-serum media (Opti-MEM, Invitrogen, Carlsbad, CA) for $4 \mathrm{~h}$ at $37^{\circ} \mathrm{C}$. At $4 \mathrm{~h}$ post-transfection, the cells were fixed with $4 \%$ paraformaldehyde and stained with a nuclear dye (Hoechst 33342, Molecular Probes, Carlsbad, CA). Imaging was performed with a confocal microscope (LSM 510 Meta, Carl Zeiss, Thornwood, NY) using the multi-track mode. Corresponding pixels of 12-bit images from each individual QD525, Cy3, or Cy5 channel were used to calculate FRET efficiencies according to Eqs. (1) and (2) and presented as a pseudo-color image and scale. The background values for $E_{12}$ were found to be $<0.1$ and $E_{23}$ to be negligible. See Supporting Information for full details on image acquisition and assessing transfection efficiency.

\section{Supporting information available}

Supplementary methods and figures; QD/BOBO/Cy5 spectra; concentration dependent quenching of $\mathrm{QD}$ by free $\mathrm{BOBO}$; DNA retention of $\mathrm{QD} / \mathrm{BOBO} / \mathrm{Cy} 5$-chitosan nanocomplexes; schematic of three-color single molecule detection setup; spectra of intact and disrupted QD/Cy3/Cy5-chitosan nanocomplexes.

\section{Acknowledgments}

This work was supported by NIH EB002849, NSF DBI-0552063, CBET0546012, and NIH DK068399.

\section{Appendix A. Supplementary data}

Supplementary data associated with this article can be found, in the online version, at doi:10.1016/j.plantsci.2004. 08.011 .

\section{References}

[1] D. Putnam, Nat. Mater. 5 (2006) 439.

[2] D.V. Schaffer, N.A. Fidelman, N. Dan, D.A. Lauffenburger, Biotechnol. Bioeng. 67 (2000) 598.

[3] D. Lechardeur, K.J. Sohn, M. Haardt, P.B. Joshi, M. Monck, R.W. Graham, B. Beatty, J. Squire, H. O'Brodovich, G.L. Lukacs, Gene Ther. 6 (1999) 482. 
[4] H. Pollard, G. Toumaniantz, J.L. Amos, H. Avet-Loiseau, G. Guihard, J.P. Behr, D. Escande, J. Gene Med. 3 (2001) 153.

[5] T. Kiang, J. Wen, H.W. Lim, K.W. Leong, Biomaterials 25 (2004) 5293.

[6] M. Koping-Hoggard, K.M. Varum, M. Issa, S. Danielsen, B.E. Christensen, B.T. Stokke, P. Artursson, Gene Ther. 11 (2004) 1441.

[7] H.Q. Mao, K.W. Leong, Adv. Genet. 53 (2005) 275.

[8] K. Itaka, A. Harada, K. Nakamura, H. Kawaguchi, K. Kataoka, Biomacromolecules 3 (2002) 841.

[9] H.J. Kong, J. Liu, K. Riddle, T. Matsumoto, K. Leach, D.J. Mooney, Nat. Mater. 4 (2005) 460.

[10] K. Raemdonck, K. Remaut, B. Lucas, N.N. Sanders, J. Demeester, S.C. De Smedt, Biochemistry 45 (2006) 10614.

[11] K. Remaut, B. Lucas, K. Raemdonck, K. Braeckmans, J. Demeester, S.C. De Smedt, Biomacromolecules 8 (2007) 1333.

[12] A.R. Clapp, I.L. Medintz, J.M. Mauro, B.R. Fisher, M.G. Bawendi, H. Mattoussi, J. Am. Chem. Soc. 126 (2004) 301.

[13] I.L. Medintz, H.T. Uyeda, E.R. Goldman, H. Mattoussi, Nat. Mater. 4 (2005) 435

[14] C.Y. Zhang, H.C. Yeh, M.T. Kuroki, T.H. Wang, Nat. Mater. 4 (2005) 826.

[15] Y.P. Ho, H.H. Chen, K.W. Leong, T.H. Wang, J. Control Rel. 116 (2006) 83.

[16] H.H. Chen, Y.P. Ho, X. Jiang, H.Q. Mao, T.H. Wang, K.W. Leong, Mol. Ther. 16 (2008) 324.

[17] I.L. Medintz, A.R. Clapp, H. Mattoussi, E.R. Goldman, B. Fisher, J.M. Mauro, Nat. Mater. 2 (2003) 630.

[18] E. Haustein, M. Jahnz, P. Schwille, Chemphyschem 4 (2003) 745.

[19] S. Wang, B.S. Gaylord, G.C. Bazan, J. Am. Chem. Soc. 126 (2004) 5446.

[20] E. Galperin, V.V. Verkhusha, A. Sorkin, Nat. Methods 1 (2004) 209.

[21] K. Roy, H.Q. Mao, S.K. Huang, K.W. Leong, Nat. Med. 5 (1999) 387.

[22] X. Jiang, H. Dai, C.Y. Ke, X. Mo, M.S. Torbenson, Z. Li, H.Q. Mao, J. Control Rel. 122 (2007) 297.

[23] H.M. Watrob, C.P. Pan, M.D. Barkley, J. Am. Chem. Soc. 125 (2003) 7336

[24] T.C. Lim, V.J. Bailey, Y.P. Ho, T.H. Wang, Nanotechnology 19 (2008) 075701.

[25] H.Q. Mao, K. Roy, V.L. Troung-Le, K.A. Janes, K.Y. Lin, Y. Wang, J.T. August, K.W. Leong, J. Control Rel. 70 (2001) 399.

[26] K. Remaut, B. Lucas, K. Braeckmans, N.N. Sanders, S.C. De Smedt, J. Demeester, J. Control Rel. 103 (2005) 259.

[27] T.H. Wang, Y. Peng, C. Zhang, P.K. Wong, C.M. Ho, J. Am. Chem. Soc. 127 (2005) 5354.

[28] S. Hohng, C. Joo, T. Ha, Biophys. J. 87 (2004) 1328.

[29] N. Shimizu, F. Kamezaki, S. Shigematsu, Nucl. Acids Res. 33 (2005) 6296.

[30] J.P. Clamme, A.A. Deniz, Chemphyschem 6 (2005).

[31] E. Galperin, V. Verkhusha, A. Sorkin, Nat. Methods 1 (2004).

[32] E.A. Jares-Erijman, T.M. Jovin, Nat. Biotechnol. 21 (2003) 1387.

[33] H. Dai, X. Jiang, G.C. Tan, Y. Chen, M. Torbenson, K.W. Leong, H.Q. Mao, Int. J. Nanomed. 1 (2006) 507.

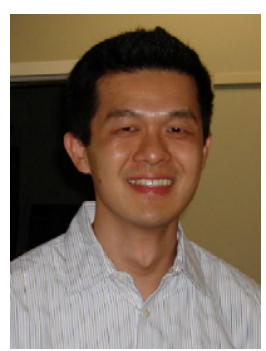

Dr. Hunter $\mathbf{H}$. Chen is currently working in formulation development for a biotechnology company. He received his $\mathrm{PhD}$ and MSE degrees in Biomedical Engineering from Johns Hopkins University where his doctoral dissertation, under the mentorship of Dr. Kam W. Leong, was focused on developing and applying nanophotonic methods to monitor the intracellular trafficking and unpacking of DNA nanocomplexes. His Master's thesis work involved optical and MR image guided gene and drug delivery. He received his $B S$ in Bioengineering from the University of California at Berkeley.

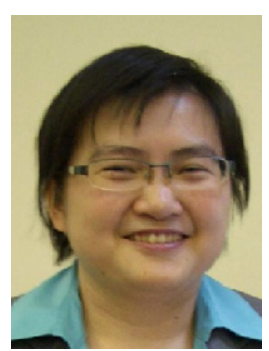

Dr. Yi-Ping Ho received her BS and MS in Power Mechanical Engineering from National TsingHua University, Taiwan and PhD in Mechanical Engineering from Johns Hopkins University, respectively. She is currently a Postdoctoral Research Associate at Duke University in the Department of Biomedical Engineering. Her research focuses on integrating nanophotonics, novel molecular constructs and microfluidics for detecting nucleic acids in the context of disease diagnostics and gene therapy.

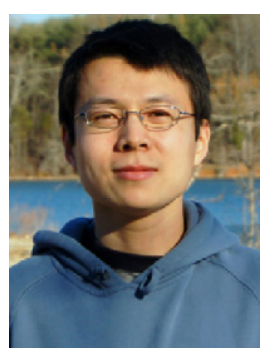

Mr. Xuan Jiang was born in Luoyang, China in 1979. He received his BS degree in polymer science and engineering from Fudan University in 2001 and MS degree in Chemistry from National University of Singapore in 2004. Currently, he is a 4th-year PhD student in the Department of Materials Science and Engineering at Johns Hopkins University. His research focuses on the development of polymeric micelle systems for liver-targeted gene delivery. He has co-authored 9 peer-reviewed journal publications.

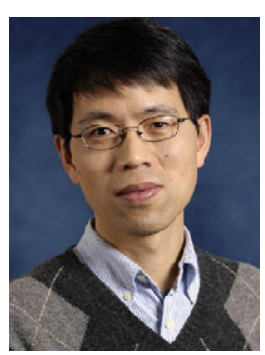

Dr. Hai-Quan Mao received his BS and PhD in polymer chemistry from Wuhan University in 1988 and 1993, respectively. He completed his postdoctoral research in the Department of Biomedical Engineering at Johns Hopkins University from 1995 to 1998 , and moved to Johns Hopkins in Singapore as a research investigator. He also taught at the Department of Materials Science in the National University of Singapore as an adjunct assistant professor from 2001 to 2003. Since 2003, he has been an assistant professor in Materials Science and Engineering at the Johns Hopkins University. Dr. Mao received the Capsugel Award for Outstanding Research from the Controlled Release Society in 1998, and the Faculty Early Career Award from the National Science Foundation in 2008. He has co-authored 66 peer-reviewed journal publications and 16 US patents. His current research focuses on engineering polymeric nanomaterials for gene delivery and for regenerative medicine applications.

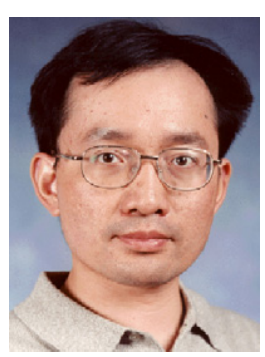

Prof. Wang received his BS and MS in Mechanical Engineering from National Taiwan University in 1988 and 1994. He served in the R.O.C. military service from 1994 to 1996 and then worked in Taiwan Semiconductor Manufacturing Company (TSMC) from 1996 to 1998. He received his $\mathrm{PhD}$ in Mechanical Engineering from University of California, Los Angeles in 2002. After graduation, Dr. Wang joined The Johns Hopkins University as a faculty member in the departments Mechanical Engineering and Biomedical Engineering. His research areas includes single molecule detection, nano-biomolecular sensors and microfluidics and the their applications to molecular diagnostics. Prof. Wang received the NSF CAREER award in 2006. 


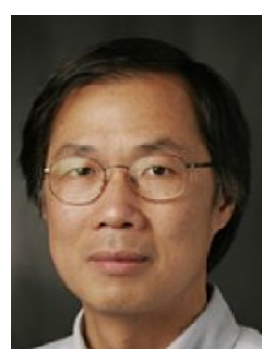

Dr. Kam Leong is the James B. Duke Professor of Biomedical Engineering at Duke University. He received his PhD in Chemical Engineering from the University of Pennsylvania and a postdoctoral training in Applied Biological Sciences at MIT. After serving as a faculty in the Department of Biomedical Engineering at The Johns Hopkins School of Medicine for 20 years, he moved to Duke University in 2006 to lead a research initiative on applying nanotechnology to drug, gene, immuno-, and cell therapy. In addition to being a research faculty at the Duke-NUS Graduate Medical School in Singapore, he also holds a Distinguished Visiting Professorship at the National University of Singapore. He serves on the editorial boards of eight journals, owns more than 35 issued patents, and has published $\sim 200$ peer-reviewed research manuscripts. The major research areas of his laboratory involve applying nanoparticles for gene delivery and understanding the response of stem cells to nanotopographical cues for stem cell tissue engineering. 